A Formula in the Theory of Single Theta-Functions. By A. C. Dixon. Communicated February 8th, 1900. Received, iu revised form, April 16th, 1900.

The addition-theorem for three arguments in the case of the functions sn $u, \operatorname{cn} u, \operatorname{dn} u$ is perhaps most compactly given by the formula

$$
\begin{aligned}
H u_{1} H u_{2} H u_{8} H u_{4}-\theta u_{1} & \theta u_{2} \Theta u_{3} \theta u_{4} \\
& -H\left(u_{1}+K\right) H\left(u_{2}+K\right) H\left(u_{8}+K\right) H\left(u_{4}+K\right) \\
& +\theta\left(u_{1}+K\right) \theta\left(u_{3}+K\right) \theta\left(u_{3}+K\right) \Theta\left(u_{4}+K\right)=0,
\end{aligned}
$$

where $u_{1}, u_{2}, u_{3}, u_{4}$ are any four arguments whose sum. is zero. For, by such substitutions as

$$
u_{1}+K, u_{2}-K, u_{3}, u_{4} \text { for } u_{1}, u_{3}, u_{3}, u_{4},
$$

other formuloe may be derived from this one, and thus the sn, cn, dn of $u_{4}$ expressed in terms of those of $u_{1}, u_{2}, u_{8}$ in many ways.

For the functions

$$
H u: H\left(u+\frac{4}{3} K\right): H\left(u+\frac{8}{3} K\right)
$$

there is a corpesponding theorem, namely, that, if $u_{1}+u_{2}+u_{3}=0$, then

$$
\begin{aligned}
H u_{1} H u_{2} H u_{3}+H\left(u_{1}+\frac{4}{3} K\right) H\left(u_{9}+\frac{4}{3} K\right) H\left(u_{3}+\frac{4}{3} K\right) \\
+H\left(u_{1}+\frac{8}{3} K\right) H\left(u_{2}+\frac{8}{3} K\right) H\left(u_{3}+\frac{8}{3} K\right)=0 .
\end{aligned}
$$

[Quarterly Journal, Vol. xxiv., p. 181 (37a), p. 225 (76).]

Again, if $u_{1}, u_{3}, \ldots, u_{n}$ are $u$ angles whose sum is $\frac{\pi}{2}$,

$$
\Pi \cos u-\Pi \cos \left(u+\frac{\pi}{n}\right)+\Pi \cos \left(u+\frac{2 \pi}{n}\right) \ldots \text { to } n \text { terms }=0 \text {. }
$$

These are instances of a general theorem which it is the object of this note to investigate, that, if $\theta u$ is a holomorphic function of $u$, such that

$$
\begin{aligned}
& \theta(u+2 \omega)=e^{a u+\beta} \theta u, \\
& \theta\left(u+2 \omega^{\prime}\right)=e^{\alpha^{\prime} u+\beta^{\prime}} \theta u,
\end{aligned}
$$

and having only a simple zero within the parallelogram of the 
periods $2 \omega ; 2 \omega^{\prime}$, namely, when $u=0$, and if $\theta_{r,} u$ denotes

$$
\begin{aligned}
\exp \left\{-\left(\frac{r a}{m}+\frac{s a^{\prime}}{n}\right)\left(u+\frac{r \omega}{m}+\frac{s \omega^{\prime}}{n}\right)+\frac{r}{m}(\alpha \omega-\beta)+\right. & \left.\frac{s}{n}\left(a^{\prime} \omega^{\prime}-\beta^{\prime}\right)\right\} \\
& \times \theta\left(u+\frac{2 r \omega}{m}+\frac{2 s \omega^{\prime}}{n}\right)
\end{aligned}
$$

where $r, s, m, n$ are any integer's, $m, n$ being positive, then

$$
\sum_{r=0}^{m-1} \sum_{i=0}^{n-1}(-1)^{r s+r i(m-1)+m(n-1)} \prod_{i=1}^{m n} \theta_{r s} u_{i}=0
$$

if $u_{1}, u_{2}, \ldots, u_{m n}$ are $m n$ arguments whose sum

$$
\equiv(m n-1)\left(n \omega+m \omega^{\prime}\right) \quad\left(\bmod 2 n \omega, 2 m \omega^{\prime}\right) .
$$

This formula contains implicitly the addition-theorem for $m n-1$ arguments relating to the functions of the type

$$
\theta_{r .} u / \theta u
$$

which are so important in the theory of transformation.

When $m=n=2$ it is possible to express.

$$
\sum_{r=0}^{m-1} \sum_{i=0}^{n-1}(-1)^{n+m+m(m-1)+8 m(n-1)} \prod_{i=1}^{m n} \theta_{r, d} u_{i}
$$

as the product of factors each a theta-function. This does not appear. to be possible in general. It can be done when $n=1, m=2$, and for a particular value of the modulus when $n=1, m=3$, and, again, for the trigonometrical case given above for any value of $n$. The form of the results, especially when $m n=3$, seems to show that, they are exceptional.

\section{Proof of the Formula.}

Taking periods $2 \omega, 2 \omega^{\prime}$, let $\theta u$ be a theta-function, having only a simple zero within the period parallelogram, numely, for the value 0 of the argument, and such that

$$
\begin{aligned}
& \theta(u+2 \omega)=e^{u u+\beta} \theta u, \\
& \theta\left(u+2 \omega^{\prime}\right)=e^{\alpha^{\prime} u+\beta^{\prime}} \theta u .
\end{aligned}
$$

Then it is known that $a \omega^{\prime}-a^{\prime} \omega=i \pi$, , being the sign of the imaginary part of $\omega^{\prime} / \omega$.

Consider the function

$$
\phi_{r \Delta} u \equiv e^{-\left(r a / m+s a^{\prime} / n\right) u} \frac{\theta\left(u+\frac{2 r \omega}{n}+\frac{2 s \omega^{\prime}}{n}\right)}{\theta\left(\frac{2 \gamma \omega}{m}+\frac{2 s \omega^{\prime}}{u}\right)},
$$


where $m, n, r, s$ are any four integers, the first two of which will be supposed positive and kept fixed throughout the discussion; in the exceptional case when $\frac{r}{m}, \frac{s}{n}$ are both integral, we shall suppose the denominator of $\phi_{\mathrm{ra}} u$ to be $\theta^{\prime}\left(\frac{2 r \omega}{m}+\frac{2 \delta \omega^{\prime}}{n}\right)$; similarly in like cases throughout. It is then easily found that

$$
\begin{aligned}
& \phi_{r+m, a} u=\phi_{r,} u, \\
& \phi_{r, a+n} u=\phi_{r,} u,
\end{aligned}
$$

so that there are only $m n$ functions such as $\phi_{r,} u$.

Also

$$
\begin{aligned}
& \frac{\phi_{r s}(u+2 \omega)}{\theta(u+2 \omega)} \div \frac{\phi_{r, a} u}{\theta u} \\
& =\exp \left\{-\left(\frac{r a}{m}+\frac{\delta a^{\prime}}{n}\right) 2 \omega+\alpha\left(u+\frac{2 r \omega}{m}+\frac{2 s \omega^{\prime}}{n}\right)+\beta-(\alpha u+\beta)\right\} \\
& \quad=\exp \frac{2 s \iota \pi}{n} ;
\end{aligned}
$$

and, in like manner,

$$
\frac{\phi_{r a}\left(u+2 \omega^{\prime}\right)}{\theta\left(u+2 \omega^{\prime}\right)} \div \frac{\phi_{r,} u}{\theta u}=\exp \left(-\frac{2 r u \pi}{m}\right)
$$

Thus the function $\frac{\phi_{r} u}{\theta u}$ has the two periods $2 n \omega$ and $2 m \omega^{\prime}$, and within its parallelogram the $m n$ poles

$$
2 i \omega+2 j \omega^{\prime} \quad(i=0,1, \ldots, n-1 ; j=0,1, \ldots, n-1) ;
$$

the same applies to the more general function

$$
\sum_{r=0}^{m-1} \sum_{i=0}^{n-1} A_{r s} \phi_{r s} u \div \theta u
$$

in which the coefficients $A$ are any constants. This function has therefore the value zero for $m n$ values of $u$, whose sum is

$$
\sum_{i=0}^{n-1} \sum_{j=0}^{m-1}\left(2 i \omega+2 j \omega^{\prime}\right) \text {, }
$$

that is, $\quad m n(n-1) \omega+n m(m-1) \omega^{\prime}$,

this being the sum of the values of $u$ at its poles. Now the $m n$ 
coefficients $A$ may be so determined that $\Sigma \Sigma A_{r,} \phi_{r a} u$ shall vanish for $m n-1$ distinct given arguments

$$
u_{1}, u_{2}, \ldots, u_{m-1} \text {. }
$$

It will then vanish for only one more, namely, $u_{m n}$, where

$$
u_{1}+u_{2}+\ldots+u_{m n}=m n(n-1) \omega+n m(m-1) \omega^{\prime} \text {, }
$$

so that, if there are $m n$ iarguments

$$
u u_{1}, u_{2}, \ldots, u_{m n},
$$

whose sum

$$
\equiv m n(n-1) \omega+n m(m-1) \omega^{\prime} \quad\left(\bmod 2 n \omega, 2 m \omega^{\prime}\right) \text {, }
$$

the determinant of the $m^{2} n^{8}$ quantities

$$
\phi_{1,} u_{n}
$$

must vanish; and, conversely, if this determinant vanishes, the sum of the arguments is as stated. We may write this determinant as

$$
\Sigma\left( \pm \prod_{h=1}^{m n} \phi_{r_{h} s_{h}} u_{h}\right)
$$

Now, we may put $u_{i}+2 k \omega, u_{j}-2 k \omega$ for $u_{i}, u_{j}$ without affecting the sum of the argurnents; the determinant thus becomes

$$
e^{k a\left(u_{i}-u_{j}+2 k \omega\right)} \Sigma\left\{ \pm e^{\left(s_{i}-\delta_{j}\right) 2 k \hbar \pi / n} \prod_{h=1}^{m n} \phi r_{h} s_{h} u_{h}\right\},
$$

which must vanish for the values $0,1,2, \ldots, n-1$ of $k$. From the $n$ equations thus derived it follows that the sum of those terms in the determinant for which $s_{i}-s_{j}(\bmod n)$ has any particular value must vanish separately. Any one of the parts into which the whole expression is thus divided may now be treated similarly by picking out any two arguments, increasing the one by a multiple of $2 \omega$ or $2 \omega^{\prime}$, and diminishing the other by an equal quantity. In this way the determinant is separated into parts, each of which must vanisli separately; in each part all the differences such as $s_{i}-s_{j}, r_{i}-r_{j}$ alc the same (mod $n, m$ respectively) in each term, and, using a double suffix notation for the arguments, we have the following lesult:-

$$
\sum_{r=0}^{m-1} \sum_{i=0}^{n-1}\left( \pm \prod_{i=0}^{n-1} \prod_{j=0}^{n-1} \phi_{i+r, j+i} u_{i, j}\right)=0 \text {. }
$$

It is now possible to give the proper sign to each term of the expression; an addition of 1 to $r$ causes cyclic interchanges in $n$ groups of $m$ factors each, and thus changes the sign $u(n-1)$ times; ly voL, XXX11.-No. 714 . 
symmetry, an addition of 1 to 8 changes the sign $m(n-1)$ times. Thus the result is

$$
\underset{r}{\sum}(-1)^{m(m-1)+\infty m(n-1)} \prod_{i, j} \phi_{i+r, j+d} u_{i, j}=0 .
$$

In this equation put

$$
u_{i j}=v_{j}-\frac{2 i}{m} \omega-\frac{2 j}{n} \omega^{\prime},
$$

so that

$$
\begin{aligned}
&{\underset{i, j}{\mathbb{S}} v_{i j}} \equiv \sum_{i, j} u_{i j}+n(m-1) \omega+m(n-1) \omega^{\prime} \\
& \equiv n(m n-1) \omega+m(m n-1) \omega^{\prime} .
\end{aligned}
$$

$$
\begin{gathered}
\text { Now } \frac{\phi_{i+r_{1} J+1}\left(v-\frac{2 i}{m} \omega-\frac{2 j \omega^{\prime}}{n}\right)}{\phi_{r,} v} \\
=\exp \left\{\left(\frac{r a}{m}+\frac{\delta a^{\prime}}{n}\right) v-\left(\frac{i+r}{m} a+\frac{j+\varepsilon}{n} a^{\prime}\right)\left(v-\frac{2 i}{m} \omega-\frac{2 j \omega^{\prime}}{n}\right)\right\} \\
\times \theta\left(\frac{2 r \omega}{m}+\frac{2 s \omega^{\prime}}{n}\right) \div \theta\left(2 \frac{i+r}{n} \omega+2 \frac{j+s}{n} \omega^{\prime}\right) .
\end{gathered}
$$

Thus $\Pi e^{\left(i a / m+j \alpha^{\prime} / n\right)\left(v_{i j}-(2 i \omega / m)-\left(2 j \omega^{\prime} / n\right)\right]} \phi_{i+r, j+a}\left(v_{j}-\frac{2 i}{m} \omega-\frac{2 j}{n} \omega^{\prime}\right)$

$$
=\prod_{i, j} \phi_{r,} v_{j}
$$

$$
\times \frac{\left\{\theta\left(\frac{2 r \omega}{m}+\frac{2 s \omega^{\prime}}{n}\right)\right\}^{m \omega}}{\prod_{i, j} \theta\left(2 \frac{i+r}{m} \omega+2 \frac{j+\varepsilon}{n} \omega^{\prime}\right)} \exp \left[\left(\frac{r a}{m}+\frac{s a^{\prime}}{n}\right)\left\{n(m-1) \omega+m(n-1) \omega^{\prime}\right\}\right] .
$$

To compare the denominators in expressions of this type, we have

$$
\begin{aligned}
\prod_{i, j} \theta\left(2 \frac{i+r}{m} \omega+2 \frac{j+s}{n} \omega^{\prime}\right) \div \prod_{i, j} \theta\left(2 \sum_{m}^{i} \omega+2 \frac{j+s}{n} \omega^{\prime}\right) \\
=\prod_{i=0}^{r-1} \prod_{j=0}^{n-1} \frac{\theta\left(2 \frac{i+m}{m}-\omega+2 \frac{j+s}{n} \omega^{\prime}\right)}{\theta\left(2 \frac{i}{m} \omega+2 \frac{j+8}{n} \omega^{\prime}\right)} \\
=\exp \sum_{i=0}^{r-1} \sum_{j=0}^{n-1}\left(\frac{2 i}{m} a \omega+2 \frac{j+s}{n} a \omega^{\prime}+\beta\right) \\
=\exp \left\{\frac{n}{m}-r(r-1) a \omega+r(n-1+2 s) a \omega^{\prime}+n r \beta\right\}
\end{aligned}
$$




$$
\begin{aligned}
\prod_{i, j} \theta\left(2 \frac{i}{m} \omega+2 \frac{j+8}{n} \omega^{\prime}\right) \div \prod_{, j} \theta\left(2 \frac{i}{m} \omega+2 \frac{j}{n} \omega^{\prime}\right) \\
=\prod_{i=0}^{m-1} \prod_{j=0}^{i-1} \frac{\theta\left(2 \frac{i}{m} \omega+2 \frac{j+n}{n} \omega^{\prime}\right)}{\theta\left(\frac{2 i}{m} \omega+\frac{2 j}{n} \omega^{\prime}\right)} \\
=\exp \sum_{i=0}^{m-1} \sum_{j=0}^{i-1}\left(\frac{2 i}{m} a^{\prime} \omega+\frac{2 j}{n} \alpha^{\prime} \omega^{\prime}+\beta^{\prime}\right) \\
=\exp \left\{s(m-1) \alpha^{\prime} \omega+\frac{m}{n} s(s-1) \alpha^{\prime} \omega^{\prime}+m s \beta^{\prime}\right\} .
\end{aligned}
$$

Hence

$$
\begin{gathered}
\prod_{i, j} e^{\left(i a / m+j a^{\prime} / n\right)\left[v_{v}-(2 i \omega / m)-\left(2 j \omega^{\prime}\right) / n\right]} \phi_{i+r, j+s}\left(v_{v}-\frac{2 i}{m} \omega-\frac{2 j}{n} \omega^{\prime}\right) \\
\times \prod_{i, j} \theta\left(\frac{2 i}{m} \omega+\frac{2 j}{n} \omega^{\prime}\right) \div\left\{\theta\left(\frac{2 r \omega}{m}+\frac{2 s \omega^{\prime}}{n}\right)\right\}^{m n} \prod_{i, j} \phi_{r,} v_{v} \\
=\exp \left[\left(\frac{r a}{m}+\frac{s a^{\prime}}{n}\right)\left\{n(m-1) \omega+m(n-1) \omega^{\prime}\right\}-\frac{n}{m}\left(r^{s}-r\right) a \omega\right. \\
\left.-r(n-1+2 s) a \omega^{\prime}-n r \beta-s(m-1) \alpha^{\prime} \omega-\frac{m}{n} s(s-1) a^{\prime} \omega^{\prime}-m s \beta^{\prime}\right] \\
=\exp \left\{r s\left(a^{\prime} \omega-a \omega^{\prime}\right)-m n\left(\frac{r a}{m}+\frac{s a^{\prime}}{n}\right)\left(\frac{r \omega}{m}+\frac{s i \omega^{\prime}}{n}\right)\right. \\
\left.+n r(a \omega-\beta)+m s\left(a^{\prime} \omega^{\prime}-\beta^{\prime}\right)\right\} .
\end{gathered}
$$

Thus

$$
\prod_{i, j} \phi_{i+r, j+1}\left(v_{u}-\frac{2 i}{m} \omega-\frac{2 j}{n} \omega^{\prime}\right)
$$$$
=(-1)^{r \cdot} \prod_{i, s} \phi_{r s} v_{v} \times\left\{\theta\left(\frac{2 r \omega}{m}+\frac{2 s \omega^{\prime}}{n}\right)\right\}^{m m}
$$$$
\times \exp \left\{-m n\left(\frac{r a}{m}+\frac{s a^{\prime}}{n}\right)\left(\frac{r \omega}{m}+\frac{s \omega^{\prime}}{n}\right)+n r(\alpha \omega-\beta)+m s\left(\alpha^{\prime} \omega^{\prime}-\beta^{\prime}\right)\right\}
$$$$
\div \prod_{i, j} e^{\left(i a / m+j a^{\prime} / n\right)\left[v_{i j}-(2 i \omega / m)-\left(2 j \omega^{\prime} / n\right)\right]} \theta\left(\frac{2 i}{m} \omega+\frac{2 j}{n} \omega^{\prime}\right) \text {. }
$$

The part of this expression after the sign $\div$ is common to all the terms, since it does not contain $r$ or $s$; it may therefore be discarded 
If, then, we write $\theta_{r s} v$ for

$\phi_{m,} v \theta\left(\frac{2 r \omega}{m}+\frac{2 s \omega^{\prime}}{n}\right) \exp \left\{-\left(\frac{r \alpha}{m}+\frac{s a^{\prime}}{n}\right)\left(\frac{r \omega}{m}+\frac{s \omega^{\prime}}{n}\right)+\frac{r}{m}(\alpha \omega-\beta)\right.$

that is, for

$$
\left.+\frac{s}{n} \cdot\left(\alpha^{\prime} \omega^{\prime}-\beta^{\prime}\right)\right\}
$$

$\exp \left\{-\left(\frac{r a}{m}+\frac{s \alpha^{\prime}}{n}\right)\left(v+\frac{r \omega}{m}+\frac{s \omega^{\prime}}{n}\right)+\frac{r}{n}(\alpha \omega-\beta)+\frac{s}{n}\left(\alpha^{\prime} \omega^{\prime}-\beta^{\prime}\right)\right\}$

$$
\times \theta\left(v+\frac{2 r \omega}{m}+\frac{2 s \omega^{\prime}}{n}\right)
$$

we have, finally,

$$
\sum_{r=0}^{m-1} \sum_{i=0}^{n-1}(-1)^{r s+1 n(m-1)+8 m(n-1)} \prod_{i=1}^{m n} \theta_{r s} v_{i}=0,
$$

if $v_{1}, v_{2}, \ldots, v_{m}$ are $m n$ arguments whose sum

$$
\equiv(m n-1)\left(n \omega+m \omega^{\prime}\right) \quad\left(\bmod 2 n \omega, 2 m \omega^{\prime}\right) .
$$

\section{Resolution into Factors.}

Let $F_{m n}\left(v_{1}, v_{2}, \ldots, v_{m n}\right)$ denote the expression

$$
\sum_{n=1}^{m-1} \sum_{i=0}^{n-1}(-1)^{r s+n(n-1)+m(n-1)} \prod_{i=1}^{m n} \theta_{r,} v_{i}
$$

In certain cases this can be expressed as the product of factors. For instance,

$$
\sum_{r=0}^{m-1}(-1)^{r} \prod_{i=1}^{m} \cos \left(v_{i}+\frac{r \pi}{m}\right)=\frac{m}{2^{m-1}} \cos \sum_{i=1}^{m} v_{i}
$$

there being in this case only one factor. Before discussing the other cases, it may be well to note that the constants $a, \beta, a^{\prime}, \beta^{\prime}$ are known to be connected by a further relation, which may be found as follows. The function $\theta u / \theta(-u)$ has neither pole nor zero within the parallelogram, and

$$
\begin{aligned}
& \frac{\theta(u+2 \omega)}{\theta(-u-2 \omega)} \div \frac{\theta u}{\theta(-u)}=e^{2(\beta-a \omega)} \\
& \frac{\theta\left(u+2 \omega^{\prime}\right)}{\theta\left(-u-2 \omega^{\prime}\right)} \div \frac{\theta u}{\theta(-u)}=e^{2\left(\beta^{\prime}-\alpha^{\prime} \alpha^{\prime}\right)} .
\end{aligned}
$$

Hence $\frac{d}{d u} \log \frac{\theta u}{\theta(-u)}$ has no pole within the parallelogram, and is 
doubly periodic; therefore it is a constant, say $2 k$, and

$$
\log \frac{\theta u}{\theta(-u)}=2 \kappa u+\lambda \text {. }
$$

Now $\theta u$ has a simple zero when $u=0$, and therefore

$$
\lim _{u=0} \frac{\theta u}{\theta(-u)}=-1 \text {, }
$$

or

$$
e^{\lambda}=-1 \text {, }
$$

so that

$$
\frac{\theta u}{\theta(-u)}=-e^{2 x u} \text {. }
$$

Hence

$$
e^{2 \kappa \omega}=-\frac{\theta \omega}{\theta(-\omega)}=-e^{-\Delta \omega+\beta},
$$

and we may put

$$
\begin{array}{ll}
\text { and we may put } & \beta=(\alpha+2 \kappa) \omega+\iota \pi ; \\
\text { in the same way, } & \beta^{\prime}=\left(a^{\prime}+2 \kappa\right) \omega^{\prime}+\iota \pi .
\end{array}
$$

Then the known result in the case $m=n=2$ may be written

$$
\begin{aligned}
F_{22}\left(v_{1}, v_{2}, v_{3}, v_{4}\right) & =-2 e^{\left(a+a^{\prime}-x\right) v_{2}+\times\left(r_{2}+r_{2}+v_{3}\right)-\left(a+a^{\prime}-4\right)\left(\omega+\omega^{\prime}\right)} \\
\times & \theta\left(\frac{v_{1}+v_{2}+v_{3}+v_{4}}{2}-\omega-\omega^{\prime}\right) \theta\left(\frac{v_{1}+v_{2}-v_{8}-v_{4}}{2}-\omega-\omega^{\prime}\right) \\
\times & \theta\left(\frac{v_{1}-v_{2}+v_{3}-v_{4}}{2}-\omega-\omega^{\prime}\right) \theta\left(\frac{v_{1}-v_{3}-v_{3}+v_{4}}{2}-\omega-\omega^{\prime}\right) .
\end{aligned}
$$

From this may be derived the expression in the case $m=2, n=1$, by writing $\omega^{\prime}$ for $v_{8}, \omega+\omega^{\prime}$ for $v_{4}$. Thus

$$
\begin{aligned}
\theta \omega^{\prime} \theta\left(\omega+\omega^{\prime}\right) & F_{21}\left(v_{1}, v_{2}\right) \\
=-2 e^{(a+4 x) \omega^{\prime}} \theta \frac{v_{1}+v_{3}-\omega}{2} \theta \frac{v_{1}+v_{2}+\omega}{2} & \theta\left(\frac{v_{1}-v_{2}+\omega}{2}-\omega^{\prime}\right) \\
& \times \theta\left(\frac{v_{2}-v_{1}+\omega}{2}-\omega^{\prime}\right) .
\end{aligned}
$$

In the case $m=3, n=1, F_{31}$ can be expressed as the product of factors if $\omega^{\prime} / \omega$ has the special value $\frac{1}{3}(\rho-1)$, where $\rho=e^{\hat{q} \imath \pi}$, so that

$$
\omega^{\prime}=\rho\left(\omega+\omega^{\prime}\right) \text {. }
$$

For, let $\theta_{1} u, \theta_{2} u$ now denote

$$
\begin{aligned}
& e^{-\{a(u+3 \omega)+\xi(a \omega-\beta)} \theta\left(u+\frac{2}{3} \omega\right), \\
& e^{-\{a(u+3 \omega)+\}(a \omega-\beta)} \theta\left(u+\frac{4}{3} \omega\right) .
\end{aligned}
$$

Then the functions $\theta_{1} u / \theta u, \theta_{2} u / \theta u$ have the periods $2 \omega, 6 \omega^{\prime}$, that is, 
$2 \omega, 2 \rho \omega, 2 \rho^{2} \omega$; their poles are $0, \pm 2 \omega^{\prime}$ or $0, \pm \frac{2}{3}(\rho-1) \omega$. The zeros of $\theta_{1} u / \theta u$ are $-\frac{2}{3} \omega,-\frac{2}{3} \omega \pm \frac{2}{3}(\rho-1) \omega$, that is $-\frac{2}{3} \omega,-\frac{2}{3} \rho \omega,-\frac{2}{3} \rho^{2} \omega$, and those of $\theta_{8} u / \theta u$ are $\frac{2}{3} \omega, \frac{2}{3} \omega \pm \frac{2}{3}(\rho-1) \omega$, that is $\frac{2}{3} \omega, \frac{2}{3} \rho \omega, \frac{2}{3} \rho^{2} \omega$. Hence $\theta_{1} u / \theta u$ and $\theta_{1} \rho u / \theta \rho u$ are functions of $u$ having the same periods, poles and zeros; this is evident in the case of the periods and zeros; a pole of $\theta_{1} \rho u / \theta \rho u$ is $\frac{2}{3}(\rho-1) \rho^{2} \omega$, which is equal to $\frac{2}{3}(\rho-1) \omega+2 \omega$, and therefore equivalent to a pole of $\theta_{1} u / \theta u$; similarly for the pole $-\frac{2}{3}(\rho-1) \rho^{2} \omega$. Thus $\theta_{1} \rho u / \theta \rho u$ only differs from $\theta_{1} u / \theta u$ by a constant factor which is found to be $\rho^{2}$ on giving $u$ a small value. Thus

$$
\frac{\theta_{1} \rho u}{\theta_{1} u}=\frac{\theta \rho u}{\rho \theta u},=\frac{\theta_{3} \rho u}{\theta_{2} u} \text { similarly. }
$$

Wach of the fractions in fact

$$
=\exp \left\{\frac{u^{2}}{4 \omega^{\prime}}\left(\alpha^{\prime} \rho^{2}-\alpha^{\prime} \rho-\alpha \rho\right)+\kappa u(\rho-1)\right\} .
$$

For let $\frac{\theta \rho u}{\rho \theta u} \exp \left\{\frac{u^{2}}{4 \omega^{\prime}}\left(a^{\prime} \rho-a^{\prime} \rho^{2}+a \rho\right)+\kappa u(1-\rho)\right\}=\chi(u)$.

Then

$$
\begin{aligned}
& \frac{\chi(u+2 \omega)}{x^{u}}=\frac{\theta\left(\rho u+6 \omega^{\prime}+2 \omega\right)}{\theta \rho u} \quad \begin{array}{c}
\theta u \\
\theta(u+2 \omega)
\end{array} \\
& \times \exp \left\{\frac{\omega}{\omega^{\prime}}(u+\omega)\left(a^{\prime} \rho-a^{\prime} \rho^{2}+a \rho\right)+2 \kappa \omega(1-\rho)\right\} \\
& =\exp \left\{\left(3 a^{\prime}+u\right) \rho \imath+6 a^{\prime} \omega^{\prime}+6 a^{\prime} \omega+3 \beta^{\prime}+\beta-a u-\beta\right. \\
& \left.+\left(\rho^{2}-1\right)(u+\omega)\left(\alpha^{\prime} \rho-\alpha^{\prime} \rho^{2}+\alpha \rho\right)+2 \kappa \omega(1-\rho)\right\}=1 \text {; }
\end{aligned}
$$

also

$$
\begin{aligned}
\frac{\chi\left(u+2 \omega^{\prime}\right)}{\chi^{u}}= & \frac{\theta\left(\rho u-2 \omega-4 \omega^{\prime}\right)}{\theta \rho u} \frac{\theta u}{\theta\left(u+2 \omega^{\prime}\right)} \\
& \quad \times \exp \left\{\left(u+\omega^{\prime}\right)\left(a^{\prime} \rho-a^{\prime} \rho^{2}+\alpha \rho\right)+2 \kappa \omega^{\prime}(1-\rho)\right\} \\
= & \exp \left\{-a^{\prime}\left(2 \rho u-4 \omega-6 \omega^{\prime}\right)-2 \beta^{\prime}-a(\rho u-2 \omega)-\beta-\alpha^{\prime} u-\beta^{\prime}\right. \\
& \left.+\left(u+\omega^{\prime}\right)\left(a^{\prime} \rho-\alpha^{\prime} \rho^{2}+a \rho\right)+2 \kappa \omega^{\prime}(1-\rho)\right\}=1 .
\end{aligned}
$$

These reductions depend on the relations

$$
3 \omega^{\prime}+\omega=\rho \omega, \quad \rho \omega^{\prime}=-\omega-2 \omega^{\prime}, \quad a \omega^{\prime}-a^{\prime} \omega=\iota \pi .
$$

It follows that $\chi u$ is doubly periodic, and therefore constant, since it has no poles. Its value is 1 when $u=0$, and hence the statement made is proved.

Now $F_{81}\left(v_{1}, v_{9}, v_{8}\right)=\theta v_{1} \theta v_{2} \theta v_{3}+\theta_{1} v_{1} \cdot \theta_{1} v_{3} \cdot \theta_{1} v_{3}+\theta_{2} v_{1} \cdot \theta_{2} v_{9} \cdot \theta_{2} v_{3}$. 
Hence $F_{81}\left(v_{1}, v_{9}, v_{3}\right), F_{81}\left(v_{1}, \rho v_{2}, \rho^{3} v_{8}\right), F_{31}\left(v_{1}, \rho^{2} v_{2}, \rho v_{8}\right)$ only differ by an exponential factor, and the zeros of each, as a function of $v_{1}$, are $\left(\bmod 2 \omega, 6 \omega^{\prime}\right),-v_{8}-v_{s},-\rho v_{9}-\rho^{2} v_{s},-\rho^{2} v_{2}-\rho 1_{s}$. Now

$$
\begin{aligned}
& 2 \omega=-\left(\rho-\rho^{2}\right)\left(2 \omega+4 \omega^{\prime}\right), \\
& 6 \omega^{\prime}=\left(\rho-\rho^{2}\right)\left(4 \omega+6 \omega^{\prime}\right),
\end{aligned}
$$

so that the periods $2 \omega, 6 \omega^{\prime}$ are equivalent to $\left(\rho-\rho^{2}\right) 2 \omega,\left(\rho-\rho^{2}\right) 2 \omega^{\circ}$. and the zeros and periods of $F_{\mathrm{si}}\left(v_{1}, v_{2}, v_{3}\right)$ coincide with those of

In fact, if

$$
\theta \frac{v_{1}+v_{2}+v_{8}}{\rho-\rho^{2}} \theta \frac{v_{1}+\rho v_{8}+\rho^{2} v_{8}}{\rho-\rho^{2}} \theta \frac{v_{1}+\rho^{2} v_{9}+\rho v_{8}}{\rho-\rho^{8}} \text {. }
$$

$$
\begin{aligned}
f\left(v_{1}, v_{2}, v_{3}\right)= & \theta \frac{v_{1}+v_{3}+v_{3}}{\rho-\rho^{2}} \theta \frac{v_{1}+\rho v_{2}+\rho^{2} v_{3}}{\rho-\rho^{2}} \theta \frac{v_{1}+\rho^{2} v_{2}+\rho v_{8}}{\rho-\rho^{2}} \\
& \times\left(\rho-\rho^{2}\right) \exp \left[-\frac{1}{4 \omega}\left\{2 v_{1}^{2}+\left(v_{2}+v_{3}\right)^{2}\right\}\left\{\rho a+\alpha^{\prime}\left(\rho-\rho^{2}\right)\right\}\right. \\
& \left.-\kappa\left(2 \rho^{2} v_{1}-v_{2}-v_{9}\right)\right]
\end{aligned}
$$

it may be verified without difficulty that

$$
F_{81}\left(v_{1}, v_{2}, v_{8}\right) \div f\left(v_{1}, v_{2}, v_{8}\right)
$$

is unchanged by the following substitutions for $v_{1}, v_{2}, v_{3}$, namely, $v_{1}+2 \omega, v_{2}, v_{3} ; v_{1}+6 \omega^{\prime}, v_{2}, v_{8} ; v_{1}+2 \omega, v_{2}+2 \omega, v_{8}+2 \omega ; v_{1}+2 \omega^{\prime}, v_{2}+2 \omega^{\prime}$, $v_{3}+2 \omega^{\prime} ; v_{1}, v_{3}+2 \omega, v_{8}-2 \omega ; v_{1}, v_{3}+2 \omega^{\prime}, v_{3}-2 \omega^{\prime}$. Hence $F_{31} \div f$ is a doubly periodic function of each of the three arguments, and it is never infinite. Thus it is a constant. Again, if $v_{2}=-v_{3}=\frac{2}{3} \omega$, this constant is readily seen to be 1 . Thus $F_{91}=f$, that is, when

$$
\begin{gathered}
\omega+\omega^{\prime}=\rho^{2} \omega, \\
F_{81}\left(v_{1}, v_{3}, v_{3}\right)=\left(\rho-\rho^{2}\right) \exp \left[-\frac{1}{4 \omega}\left\{2 v_{1}^{2}+\left(v_{2}+v_{8}\right)^{2}\right\}\left\{\rho a+\left(\rho-\rho^{2}\right) a^{\prime}\right\}\right. \\
\left.-\kappa\left(2 \rho^{2} v_{1}-v_{2}-v_{3}\right)\right] \\
\times \theta \frac{v_{1}+v_{8}+v_{9}}{\rho-\rho^{2}} \theta \frac{v_{1}+\rho v_{8}+\rho^{2} v_{3}}{\rho-\rho^{2}} \theta \frac{v_{1}+\rho^{2} v_{8}+\rho v_{8}}{\rho-\rho^{2}} .
\end{gathered}
$$

This result is simplified by taking $\theta u$ to. be $\sigma u$, for then $\kappa=0$, $a=a^{\prime}(\rho-1)$, and $F_{y_{1}}\left(v_{1}, v_{2}, v_{8}\right)$, that is

$$
\begin{gathered}
\sigma v_{1} \sigma v_{2} \sigma v_{3}-e^{-\ln \left(v_{1}+v_{3}+r_{2}+\omega\right)} \sigma\left(v_{1}+\frac{2}{3} \omega\right) \sigma\left(v_{2}+\frac{2}{3} \omega\right) \sigma\left(v_{3}+\frac{2}{3} \omega\right) \\
+e^{-l_{\eta}\left(v_{1}+r_{2}+r_{3}+2 \omega\right)} \sigma\left(v_{1}+\frac{4}{3} \omega\right) \sigma\left(v_{2}+\frac{4}{3} \omega\right) \sigma\left(v_{3}+\frac{4}{3} \omega\right) \\
=\left(\rho-\rho^{2}\right) \sigma \frac{v_{1}+v_{2}+v_{3}}{\rho-\rho^{2}} \sigma \frac{v_{1}+\rho v_{3}+\rho^{2} v_{3}}{\rho-\rho^{2}} \sigma \frac{v_{1}+\rho^{2} v_{2}+\rho v_{3}}{\rho-\rho^{2}} .
\end{gathered}
$$

